\title{
OPEN A novel hyperthermophilic methylglyoxal synthase: molecular dynamic analysis on the regional fluctuations
}

\author{
Gyo-Yeon $\mathrm{Seo}^{1,4}$, Hoe-Suk Lee ${ }^{3,4}$, Hyeonsoo $\mathrm{Kim}^{1}$, Sukhyeong $\mathrm{Cho}^{2}$, Jeong-Geol $\mathrm{Na}^{1}$, \\ Young Joo Yeon ${ }^{3 \varpi}$ \& Jinwon Lee ${ }^{1,2 \bowtie}$
}

Two putative methylglyoxal synthases, which catalyze the conversion of dihydroxyacetone phosphate to methylglyoxal, from Oceanithermus profundus DSM 14,977 and Clostridium difficile 630 have been characterized for activity and thermal stability. The enzyme from $O$. profundus was found to be hyperthermophilic, with the optimum activity at $80^{\circ} \mathrm{C}$ and the residual activity up to $59 \%$ after incubation of $15 \mathrm{~min}$ at $95^{\circ} \mathrm{C}$, whereas the enzyme from C. difficile was mesophilic with the optimum activity at $40^{\circ} \mathrm{C}$ and the residual activity less than $50 \%$ after the incubation at $55^{\circ} \mathrm{C}$ or higher temperatures for $15 \mathrm{~min}$. The structural analysis of the enzymes with molecular dynamics simulation indicated that the hyperthermophilic methylglyoxal synthase has a rigid protein structure with a lower overall root-mean-square-deviation value compared with the mesophilic or thermophilic counterparts. In addition, the simulation results identified distinct regions with high fluctuations throughout those of the mesophilic or thermophilic counterparts via root-mean-square-fluctuation analysis. Specific molecular interactions focusing on the hydrogen bonds and salt bridges in the distinct regions were analyzed in terms of interatomic distances and positions of the individual residues with respect to the secondary structures of the enzyme. Key interactions including specific salt bridges and hydrogen bonds between a rigid beta-sheet core and surrounding alpha helices were found to contribute to the stabilisation of the hyperthermophilic enzyme by reducing the regional fluctuations in the protein structure. The structural information and analysis approach in this study can be further exploited for the engineering and industrial application of the enzyme.

Methylglyoxal synthase (MGS, EC number 4.2.3.3) is a lyase for an efficient removal of a phosphate moiety from dihydroxyacetone phosphate (DHAP) to form pyruvaldehyde, an enol precursor that can be tautomerized spontaneously to methylglyoxal $(\mathrm{MG})^{1-4}$. DHAP and MG are intermediates in a bypass of the cellular glycolytic pathway, where MG is converted to lactate and subsequently oxidized to pyruvate. However, in vivo millimolar concentration of MG is mutagenic and interferes with de novo protein and nucleic acid synthesis ${ }^{3,5}$; hence the function of MGS is emphasized to balance the physiological concentrations of the cytotoxic product. MGS has been found and characterized from gram-negative and gram-positive bacteria such as Escherichia coli ${ }^{6}$, Pseudomonas saccharophila ${ }^{7}$, Proteus vulgaris ${ }^{8}$, Clostridium acetobutylicum ${ }^{9}$ and Bacillus subtilis ${ }^{10}$, as well as Saccharomyces cerevisiae ${ }^{11}$ and thermophilic Thermus sp. GH5 $5^{5}$.

MGS can be a valuable tool in the biochemical production of value-added chemicals from glycolytic intermediates, especially the commercial 1,2-propanediol ${ }^{12-14} .1,2$-propanediol has applications as antifreeze, a feedstock for polyester resins in film and fiber manufacture, a substrate for optically active propylene oxide and polymers, and pharmaceutical products. In previous studies, MGS-expressing E. coli was metabolically engineered to produce MG, which was then converted to 1,2-propanediol ${ }^{12,14}$. Alternatively, enzymatic synthesis of MG from DHAP can be employed considering the cytotoxicity of the product. In this case, a highly stable and active MGS is sought for the industrial applications, where harsh conditions such as high temperature are often used.

\footnotetext{
${ }^{1}$ Department of Chemical and Biomolecular Engineering, Sogang University, Seoul 04107, Republic of Korea. ${ }^{2}$ C1 Gas Refinery R\&D Center, Sogang University, Seoul 04107, Republic of Korea. ${ }^{3}$ Department of Biochemical Engineering, Gangneung-Wonju National University, Gangneung-si, Gangwon-do 25457, Republic of Korea. ${ }^{4}$ These authors contributed equally: Gyo-Yeon Seo and Hoe-Suk Lee. ${ }^{\boxplus e m a i l}$ yjyeon@gwnu.ac.kr; jinwonlee@sogang.ac.kr
} 


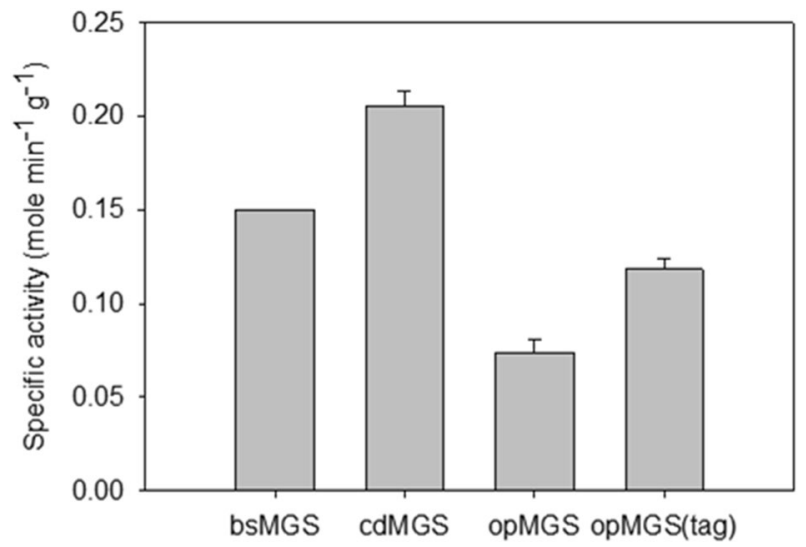

Figure 1. Specific activities of the purified MGSs at $\mathrm{pH} 7.5,30^{\circ} \mathrm{C}$. Each value and error bar represents the mean and the standard deviation of at least three independent trials, respectively. The specific activity of bsMGS from Bacillus subtilis has been reported previously and presented here for comparison ${ }^{4}$.

Advantages of the high temperature in industrial processes include improved mass transfer and reaction rates, increased substrate solubility, lower viscosity, and reduced risk of microbial contamination ${ }^{15}$. To this end, many industrial enzymes are isolated from thermophilic (optimum temperature at $50{ }^{\circ} \mathrm{C} \sim$ below $80{ }^{\circ} \mathrm{C}$ ) or hyperthermophilic (optimum temperature at $80^{\circ} \mathrm{C}$ or higher) organisms living in an extreme environment such as hot spring ${ }^{16,17}$. Furthermore, comparing the thermophilic or hyperthermophilic enzymes and the mesophilic counterparts in terms of amino acid sequences and three-dimensional structures can reveal the rationale for the increased thermostability because they share a high degree of structural similarity ${ }^{16-18}$. The rationale can be exploited to design tailor-made thermostable proteins ${ }^{19,20}$.

In this work, putative mgsA genes from Oceanithermus profundus DSM 14,977 and Clostridium difficile 630 encoding MGS enzymes were recombinantly expressed in $E$. coli, purified and characterized for optimum $\mathrm{pH}$, optimum temperatures $\left(\mathrm{T}_{\mathrm{opt}}\right)$ and thermostability, where the MGS from O. profundus was found to be hyperthermophilic. The structural basis for the activity and thermostability of the enzymes has been analyzed via molecular modeling and discussed with respect to previously characterized MGSs.

\section{Results and discussion}

Screening, expression and purification for putative MGSs. Putative $m g s A$ gene sequences were selected from the NCBI database by the sequence homology between 30 and $70 \%$ with the previously characterized MGSs such as those from Escherichia coli (ecMGS), Bacillus subtilis (bsMGS) and Thermus sp. GH5 (tsMGS). As a result, a MGS from Clostridium difficile 630 (cdMGS) with $45.3 \%$ similarity to ecMGS and a MGS from Oceanithermus profundus (opMGS) with 53.0\% similarity to tsMGS were selected for characterization after initial screening of activity. The cdMGS consists of 137 amino acids with a molecular weight of $14.1 \mathrm{kDa}$, originating from the Gram-positive, anaerobic and spore-forming Clostridium difficile strain $630^{21}$. The opMGS consists of 125 amino acids with the molecular weight of $13.9 \mathrm{kDa}$, and its gene is from the thermophilic bacterium Oceanithermus profundus isolated from a deep-sea hydrothermal vent ${ }^{22}$.

The $m g s A$ genes were cloned along with a Strep tag for affinity purification, which is known to have a minimal effect on the main protein body (See Supplementary Table S1 online) ${ }^{23,24}$. The result of protein expression, Strep-affinity purification and SDS-PAGE clearly showed purified polypeptides with appropriate sizes for cdMGS and opMGS (See Supplementary Fig. S1 online). Furthermore, a six-aspartate tag (Asp tag) was added to the $\mathrm{N}$-terminal of opMGS to increase its solubility ${ }^{25}$.

Specific activity of cdMGS and opMGS. Figure 1 shows the specific activities of the purified MGSs measured at $30{ }^{\circ} \mathrm{C}$ and $\mathrm{pH} 7.5$, along with bsMGS, a mesophilic MGS used for 1,2-propanediol synthesis with the highest known specific activity described previously ${ }^{4}$. The specific activities of cdMGS and opMGS were 0.21 and $0.07 \mathrm{~mol} \mathrm{~min}^{-1} \mathrm{~g}^{-1}$, respectively. The cdMGS had $36.7 \%$ higher specific activity than that of bsMGS with $0.15 \mathrm{~mol} \mathrm{~min} \mathrm{~m}^{-1}$. The activity of opMGS was not as high as those of bsMGS or cdMGS, but the addition of the Asp tag increased the activity to $0.12 \mathrm{~mol} \mathrm{~min}^{-1} \mathrm{~g}^{-1}$. Explaining the delicate effect of a tag on the enzymatic activity when the tag is distant from the active site remains a challenging task, although there are reports of net charge affecting the solubility and the activity of proteins ${ }^{26,27}$.

Effect of $\mathrm{pH}$ on the activity of cdMGS and opMGS. Optimal pH for the cdMGS, opMGS and opMGS (Asp tag) were investigated, which showed all three MGSs had the highest specific activity at pH 7 (Fig. 2). The cdMGS retained relatively high specific activity within the $\mathrm{pH}$ range of 5-7 but the activity decreased sharply outside the range. The optimal $\mathrm{pH}$ range was narrower for both opMGS and opMGS (Asp tag) than that for cdMGS. The three MGSs seemed to be deactivated below $\mathrm{pH} 4$ or above $\mathrm{pH}$ 8. These are comparable to the previously reported, neutral pH optima for MGSs from E. coli $(\mathrm{pH} 7.5)^{6}$, Thermus sp. GH5 (pH 6) $)^{5}$ Capra hircus (pH 


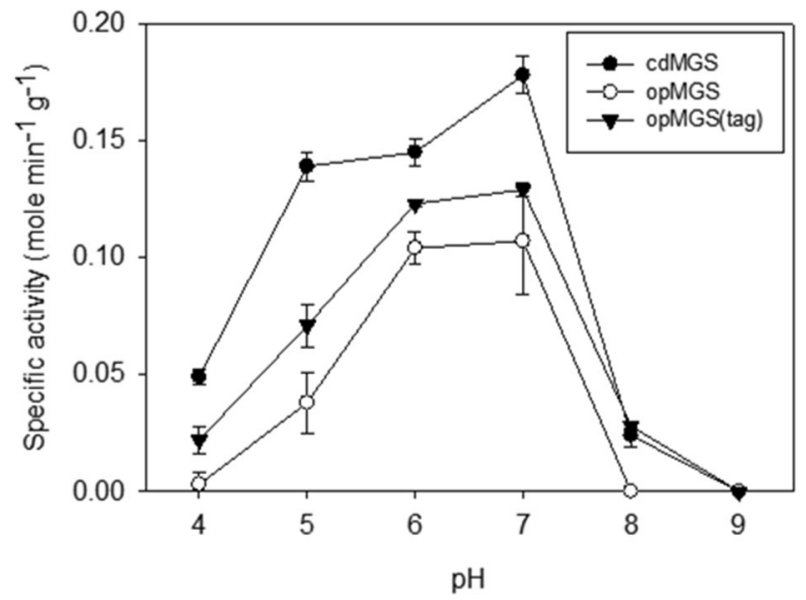

Figure 2. $\mathrm{pH}$ optima for the purified MGSs. Each value and error bar represents the mean and the standard deviation of at least three independent trials, respectively.

(a)

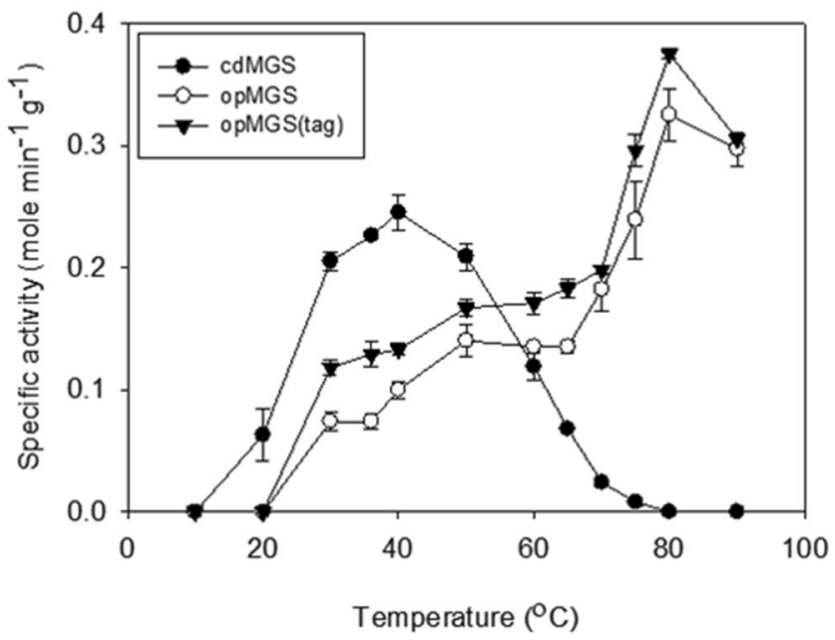

(b)

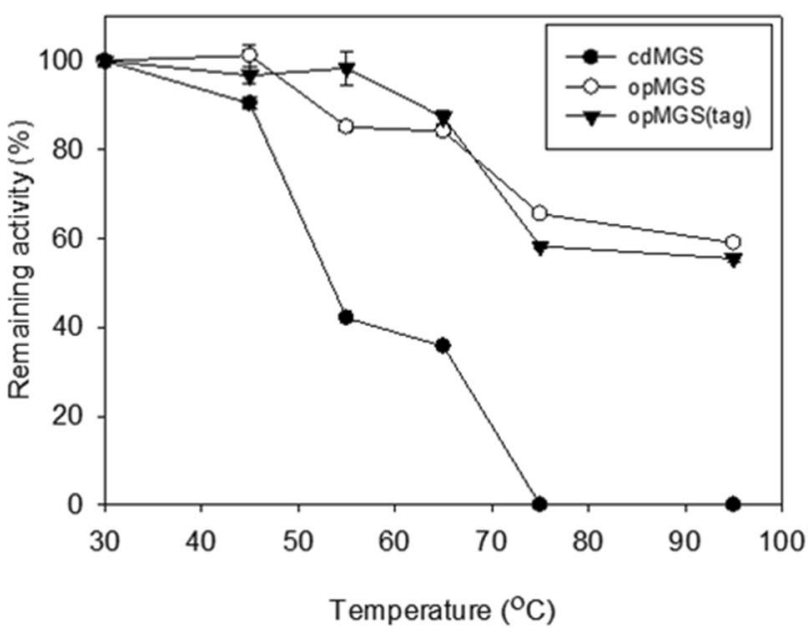

Figure 3. Thermal properties of the purified MGSs. (a) Temperature optima; (b) Thermostability. Each value and error bar represents the mean and the standard deviation of at least three independent trials, respectively.

$7.2)^{28}$, Clostridium acetobutylicum $(\mathrm{pH} 7.5)^{9}$, Proteus vulgaris $(\mathrm{pH} 7.7)^{8}$, but different from the alkalophilic MGSs from Pelomonas saccharophilia $(\mathrm{pH} 8.2)^{7}$ and Saccharomyces cerevisiae $(\mathrm{pH} 9.5-\mathrm{pH} 10.5)^{11}$.

Temperature optima and thermostability of cdMGS and opMGS. The $\mathrm{T}_{\mathrm{opt}}$ for cdMGS and opMGS were determined by carrying out the enzymatic reaction with the substrate incubated under various temperatures (Fig. 3a). The cdMGS showed the highest activity at $40^{\circ} \mathrm{C}$, while opMGS and opMGS (Asp tag) had $\mathrm{T}_{\text {opt }}$ at $80{ }^{\circ} \mathrm{C}$. The thermostability of the enzymes was also determined by incubating the enzymes at different temperatures before the activity assay (Fig. 3b). The cdMGS maintained $90 \%$ remaining activity up to $45^{\circ} \mathrm{C}$ but the activity rapidly decreaseed to less than half at $55^{\circ} \mathrm{C}$ or higher temperature. In contrast, opMGS and opMGS (Asp tag) had much higher thermal stabilities. They retained over $85 \%$ and $98 \%$ activities at $55^{\circ} \mathrm{C}$, and $59 \%$ and $55 \%$ activities at $95^{\circ} \mathrm{C}$, respectively. The results indicate opMGS has a higher thermostability than the previously studied tsMGS from Thermus sp. GH5, which had $\mathrm{T}_{\text {opt }}$ at $75^{\circ} \mathrm{C}$ and a rapidly decreasing activity over $75^{\circ} \mathrm{C}^{5}$. The tsMGS presented approximately $10 \%$ remaining activity at $95^{\circ} \mathrm{C}$. By definition, tsMGS is a moderate thermophilic enzyme, while opMGS is a hyperthermophilic enzyme with $\mathrm{T}_{\mathrm{opt}}$ at $80^{\circ} \mathrm{C}$ or higher ${ }^{16}$.

Sequence comparison of MGSs. Alignment of the amino acid sequences from the mesophilic MGSs (ecMGS, bsMGS and cdMGS) and the thermophilic MGSs (tsMGS and opMGS) indicates the catalytic function of specific residues and sequential differences responsible for the difference in the thermostability (Fig. 4). The homology between cdMGS and opMGS was $49 \%$. The thermophiles, especially the hyperthermophilic opMGS, are shorter toward the $\mathrm{C}$-terminal than the mesophilic enzymes, generating more compact structures. The active site residues identified from ecMGS and bsMGS are highly conserved among all five MGSs ${ }^{3,4}$. D71 (numbering 
ecMGS

bSMGS

cdMGS

t SMGS

ODMGS

ecMGS

bSMGS

cdMGS

t SMGS

OPMGS

ecMGS

bSMGS

cdHGS

t SMGS

OPMGS

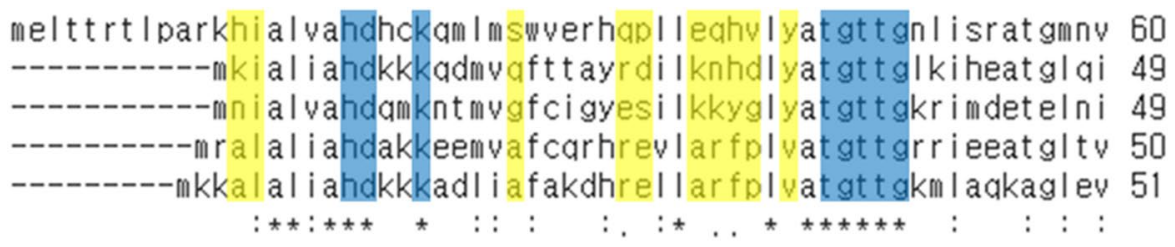

nam I sgpmggdaquga I i segki gu I i f fwdp I navphdpdvkal I r I at vwn i pvatnv 120 er fasgp I ggdaai gal i aanal d l vi f I rdp I t aaphepdvsal i r I cdvysiplat nm 109

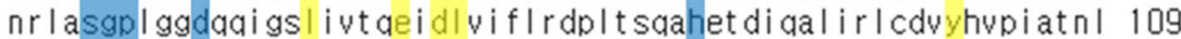
ek I I sgp I ggdagmgarvaegr i I av i f frdp I t aaphepdvaa I I rucduhgup I at np 110 ervasgp I ggd a gar i aedr i l av i f frdp I t aqphepdvsa I m r i cdvhdup I at n I 111

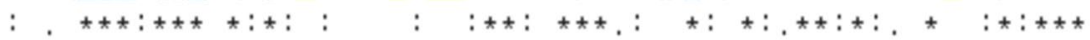

Figure 4. Amino acid sequence alignment of cdMGS and opMGS with ecMGS (E. coli), bsMGS (B. subtilis) and tsMGS (Thermus sp. GH5). The ecMGS, bsMGS and cdMGS represent mesophilic MGSs, and tsMGS and opMGS represent thermophilic MGSs. Blue-shades indicate the active site. Yellow-shades indicate residues that are found common in the thermophilic MGSs but different from the mesophilic enzymes. Asterisk, colon and full stop indicate the degree of sequential similarity from the highest to the lowest.

in ecMGS) is known to play a key catalytic role as a base to withdraw a pro-S proton from the $\mathrm{C}_{3}$ atom of DHAP, while $\mathrm{H} 19$ can stabilize the $\mathrm{O}_{3}$ hydroxyl group via hydrogen bonding. $\mathrm{H} 98$ helps the transfer of a proton to the $\mathrm{O}_{2}$ to form an enediol, although the function of $\mathrm{H} 98$ was suggested to be not critical in the catalytic mechanism itself but focused more on the regulation of the MGS conformation between active and inactive states ${ }^{29-31}$. K23, S65 and T45-G49 surround and stabilize the phosphate moiety binding via hydrogen bond and salt bridge, and G66-P67 follow the S65 in a loop covering the $\mathrm{C}_{1}$ and $\mathrm{C}_{2}$ atoms of DHAP. The residues that are present only in the thermophilic MGSs could be identified, many of which were hydrophobic residues in replacement of the hydrophilic homologues of the mesophiles. This may be used in thermostability engineering, although their specific molecular functions must be analyzed further.

Docking of DHAP in the active sites of the MGSs. The three dimensional structures of cdMGS and opMGS have been constructed by homology modeling. The active sites share a high similarity, yet the configuration of the substrate docked, DHAP, showed disaccord between the two MGSs (Fig. 5). The role of D71 in ecMGS as a catalytic base is played by D60 in cdMGS and D62 in opMGS ${ }^{30}$. The hydroxyl group at $\mathrm{O}_{3}$, oxidized to a ketone during the proton transfer, is stabilized via H8 (cdMGS) and H10 (opMGS). The H98 in ecMGS is equivalent to H87 and H89 in cdMGS and opMGS, respectively, to assist the stabilization of the $\mathrm{O}_{2}$ oxyanion and the transfer of a proton. The accessorial but not critical function is reflected in the relatively longer distance between the residue and the $\mathrm{O}_{2}$ atom than other critical catalytic distances. The backbone N from G55 (cdMGS) and G57 (opMGS) form a hydrogen bond with the phosphate oxygen where the $\mathrm{C}-\mathrm{O}$ bond is lysed. The conserved motif of TGTTG, lysine and serine (residue no. 34-38, 12 and 54 for cdMGS, and no. 36-40, 14 and 56 for opMGS) are also used to stabilise other oxygens of the phosphate group via salt bridges and hydrogen bonds. The binding energies $\left(\Delta G_{\text {bind }}\right)$ for the substrate were -3.76 and $-3.01 \mathrm{kcal} \mathrm{mol}^{-1}$ for cdMGS and opMGS, respectively, which indicated a more stable binding in the active site of cdMGS for a lower $K_{M}$.

Structural basis for the thermostability. In order to examine the molecular basis for the increased thermostability, the structures of hyperthermophilic opMGS, thermophilic tsMGS and mesophilic cdMGS have been subjected to molecular dynamics simulation (MD) of $400 \mathrm{~ns}$ at $353 \mathrm{~K}$ temperature. The overall molecular root-mean-square deviation (RMSD) was the lowest in opMGS, followed by tsMGS and cdMGS, indicating the structural rigidity was higher toward the more thermostable MGSs (Fig. 6a). In-depth residue-by-residue analysis of the structural rigidity was also carried out by calculating the root-mean-square fluctuation (RMSF) across the MGS residues (Fig. 6b). Four Regions (I-IV) of large difference in the structural rigidity among the MGSs were identified by calculating the RMSF difference between the thermophilic MGSs and cdMGS, and whether the residue RMSF difference was larger than the average RMSF difference. Each Region mainly encompasses alpha-helices, such that the Region I surrounds the alpha-helices $\alpha 1-\alpha 2$, the Region II for the $\alpha 3$ and the following loop, the Region III for the loop between $\beta 4$ and $\alpha 5$ and a half of $\alpha 5$, and the Region IV for a part of $\alpha 6$ and the following loop toward the C-terminal. These helices surround the core beta-sheet from both sides, where the mostly hydrophobic beta-sheet shows a relatively similar rigidity in the thermophilic and mesophilic MGSs. 

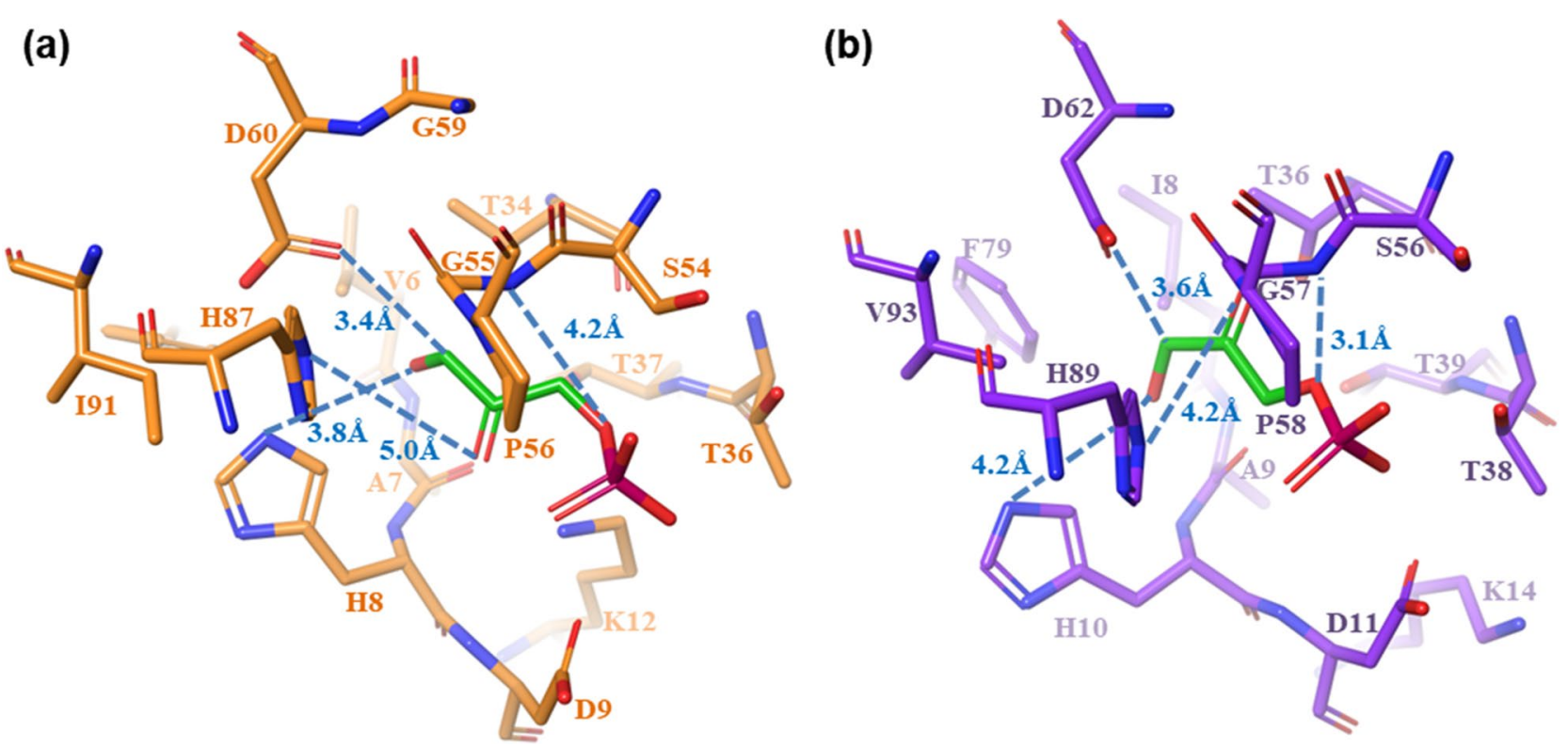

Figure 5. DHAP-docked enzymatic active sites. (a) cdMGS; (b) opMGS. The carbon atoms of DHAP, cdMGS and opMGS are colored as green, orange and purple, respectively. Nitrogen, oxygen, and phosphorous atoms are colored as blue, red and magenta, respectively. Distances between the key residues and substrate atoms are marked by dashed lines. $\Delta \mathrm{G}_{\text {bind }}$ for DHAP in cdMGS and opMGS were -3.76 and $=-3.01 \mathrm{kcal} \mathrm{mol}^{-1}$, respectively.
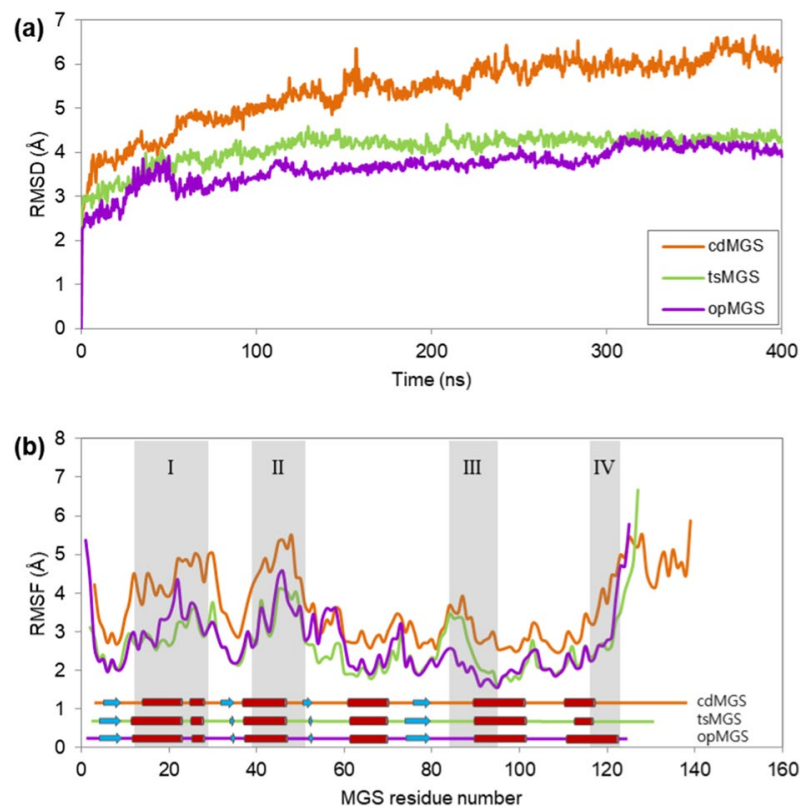

Figure 6. Molecular dynamics of opMGS, tsMGS and cdMGS. The MD simulation was carried out at $358 \mathrm{~K}$ for 400 ns. (a) Overall root-mean-square-deviation (RMSD) of the MGSs; (b) Root-mean-square-fluctuation (RMSF) for each residue of the MGSs. The three MGS sequences were aligned and a common set of residue numbers was assigned for the RMSF analysis of the homologous residues. Secondary structures such as $\alpha$-helices (red cylinders), $\beta$-strands (blue arrows) and loops (lines with corresponding colors of the MGSs) are indicated. Regions of high difference in fluctuation between cdMGS and opMGS are shown as shaded boxes (I-IV).

Several factors increasing the stability of proteins in thermophiles have been suggested previously, such as atomic packing, loop shortening and increased number of salt bridges ${ }^{32-34}$. Specific molecular interactions that could enhance the structural rigidity in the four Regions of hyperthermophilic opMGS focusing on salt bridges and hydrogen bonds were therefore analyzed. Disulfide bonding that is widely found in thermophilic archaean proteins was not found in the bacterial opMGS and tsMGS, with a limited number of cysteine residues positioned distant from each other ${ }^{35}$. It has been reported not all thermophilic organisms contain an abundance of disulfides, 
(a)

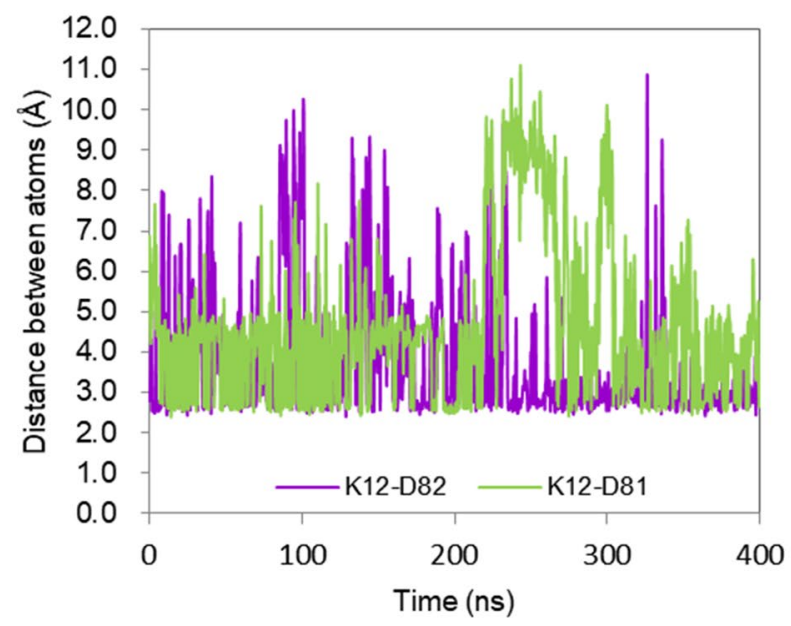

(c)

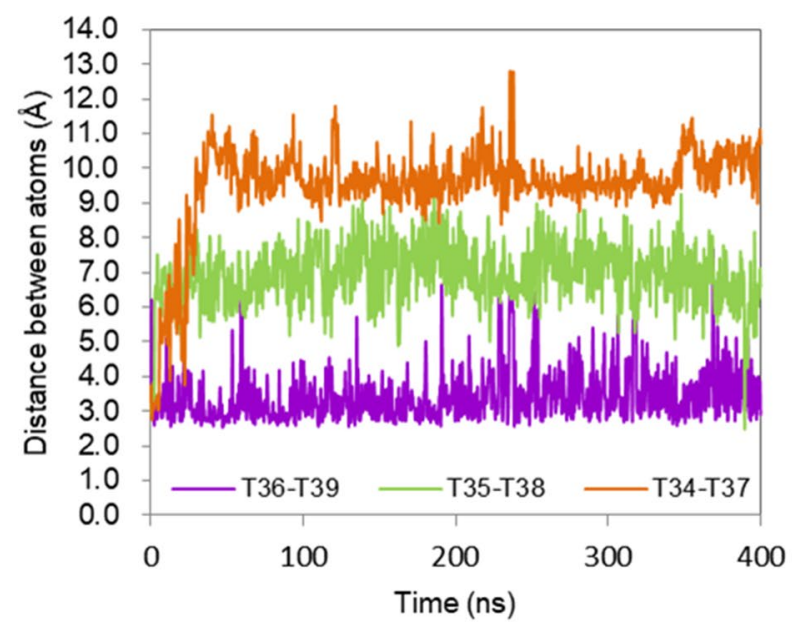

(b)

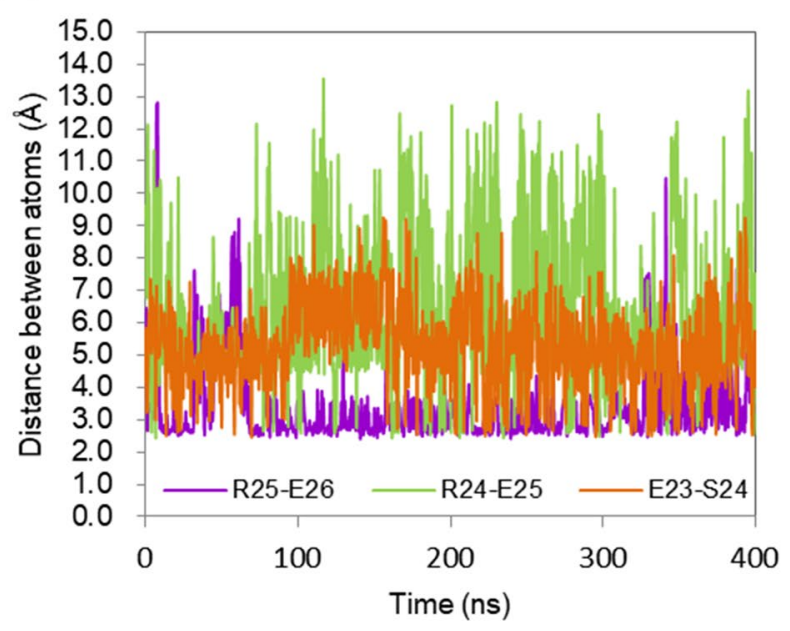

(d)

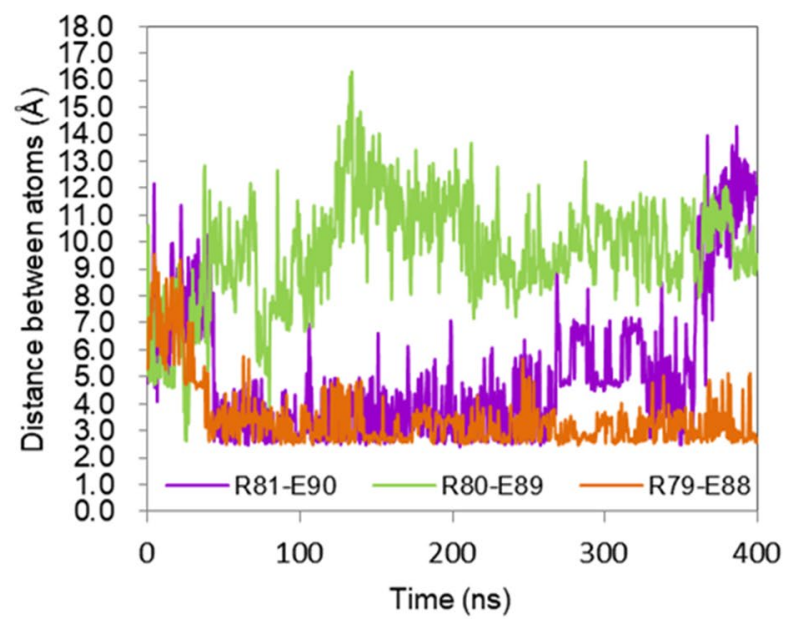

Figure 7. Interaction distance between residues that can affect RMSF in MGSs. Purple, green and orange correspond to the interactions between residues at homologous positions in opMGS, tsMGS, and cdMGS, respectively, with the residue number insets. (a) Salt bridges between specific lysines and aspartates in opMGS and tsMGS. The lysine is replaced by a methionine and cannot form a salt bridge in cdMGS; (b) Salt bridges between specific arginines and glutamates in opMGS and tsMGS. The salt bridge is replaced by a hydrogen bond between glutamate and serine in cdMGS; (c) Hydrogen bonds between specific threonines in the three MGSs; (d) Salt bridges between specific arginines and glutamates in the three MGSs.

especially the methanogens and sulfur-reducing chemolithotrophs growing at strongly reducing conditions which necessitate the preclusion of cytosolic disulfide bonding ${ }^{36}$. However, utilization of the MGSs in industry can be free from such restriction, and may be engineered with disulfides to further increase the thermal stability.

Figures 7 and 8 show the distances between residues that could form the salt bridges or hydrogen bonds. K12-D82 in opMGS can form a salt bridge and maintain them at $353 \mathrm{~K}$ in a reasonable frequency to increase the interaction of the $\alpha 1$ helix in the Region I and the loop shortly after the $\beta 4$, whereas a corresponding bridge cannot be formed by M11 and D80 or nearby residues in cdMGS (Fig. 7a). The aspartate is conserved among the five MGSs in the sequence alignment. A11 in tsMGS is at the homologous position to the K12, but the adjacent K12 side chain is within the bridge-forming distance from the aspartate. R25-E26 in opMGS or corresponding residues in tsMGS and cdMGS can form salt bridges that increase the stability of the short $\alpha 2$ helix in the Region I, but much higher frequency was observed in opMGS than in tsMGS or cdMGS (Fig. 7b). The two residues are adjacent and not involved in an inter-secondary structure interaction, but instability in the $a 2$ can lead to increased fluctuations in the $\alpha 1$ because the $\alpha 2$ is right at the end of the $\alpha 1$ in a bent conformation, holding $\alpha 1$ in place. The hydrogen bond between T36-T39 can be observed at higher frequency in opMGS, whereas the distances were longer in tsMGS and cdMGS, hardly forming the bond (Fig. 7c). Although these residues were conserved in the sequence alignment of the five MGSs, the $\alpha 3$ distorts extensively in cdMGS, leading to the increased distance (Fig. 8c). The T36-T39 hydrogen bond can increase the interaction between the $\beta 2$ and the $\alpha 3$ in the Region II, contributing to the local rigidity in opMGS. R81-E90 in opMGS and R79-E88 in cdMGS can form salt bridges between the loop following the $\beta 4$ and the $\alpha 5$ in the Region III, but the effect on the rigidity of 
(a)

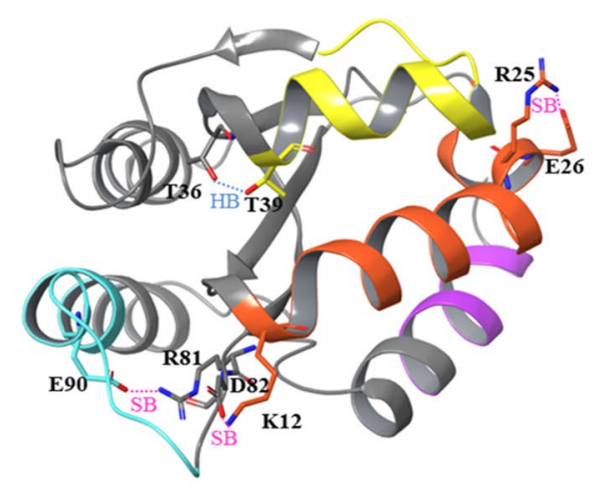

(b)

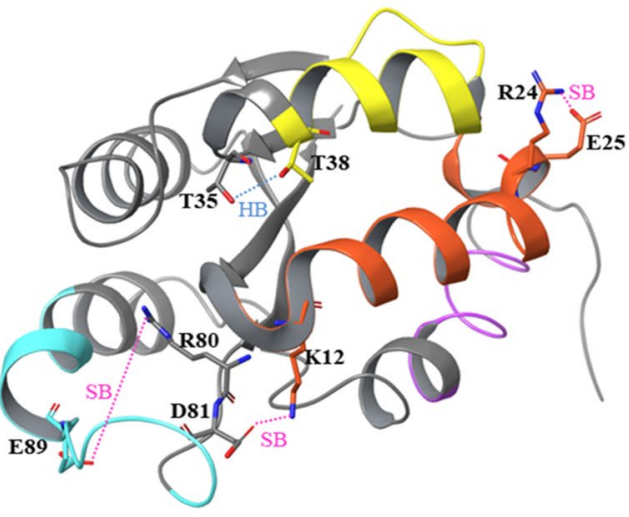

(c)

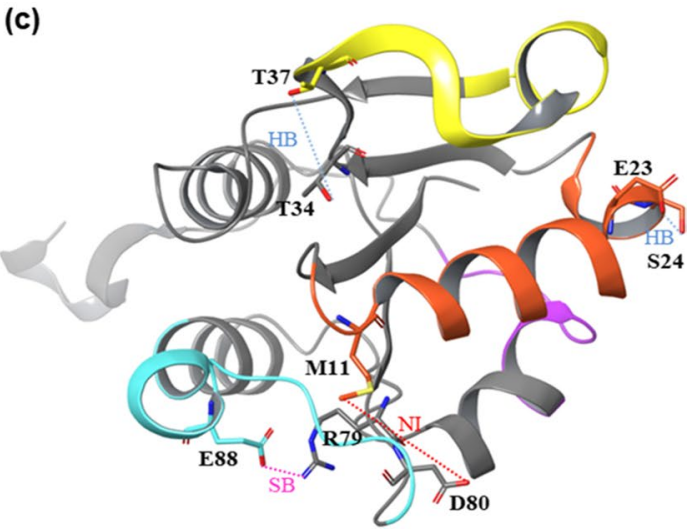

Figure 8. Modeled structures of the MGSs. (a) opMGS; (b) tsMGS; and (c) cdMGS. The regions of high RMSF difference are colored as orange (I), yellow (II), cyan (III) and purple (IV). The conformation of residues between which the distances are measured are indicated with the expected interactions such as salt bridge (SB), hydrogen bond (HB) and no corresponding interaction (NI).

the region can be different in opMGS and cdMGS because R79 in cdMGS is located in a loop with higher flexibility due to lack of an interaction between M11 and D80 (Fig. 7d). Interestingly, tsMGS could not form a salt bridge at this location that may contribute to the lowered stability than that of opMGS. In combination with the K12-D82 bridge found in both opMGS and tsMGS but not in cdMGS, it is implicated that the K12-D82 bridge is important for being a thermophile, and the R81-E90 bridge is important for being a hyperthermophile in the MGSs. Other hydrogen bonds and salt bridges have been extensively searched throughout the MGS structures and their interatomic distances measured, but no significant difference between the hyperthermophilic, thermophilic and mesophilic MGSs were observed. This includes the Region IV, and its high fluctuation in cdMGS compared to the thermophiles could be derived from its extended C-terminal loop rather than from the absence of specific molecular interactions. 
Overall, the increased thermal stability in tsMGS and opMGS could be explained by a rigid beta-sheet core and capability of alpha-helices to interact with the core via salt bridges and hydrogen bonds that specifically reduce regional fluctuations.

\section{Conclusion}

In this study, a hyperthermophilic MGS has been discovered and structurally analyzed for its high thermostability. Two bacterial methylglyoxal synthases from C. difficile 630 and O. profundus were cloned, expressed and characterized, of which cdMGS was mesophilic with $\mathrm{T}_{\text {opt }}$ at $40^{\circ} \mathrm{C}$ and opMGS was hyperthermophilic with $\mathrm{T}_{\text {opt }}$ at $80^{\circ} \mathrm{C}$. The opMGS presented the highest known $\mathrm{T}_{\text {opt }}$ and thermostability among MGSs characterized so far. These newly characterized MGSs can be added to the set of enzymes for utilization in industry to produce value-added chemicals. Furthermore, substrate docking and molecular dynamics simulation were employed to explain their activity and stability. Identification of key interactions including specific salt bridges and hydrogen bonds that restrict the regional fluctuations in MGSs can be exploited to further engineer the enzymes for industrial purposes.

\section{Methods}

Gene cloning. The mgsA genes from Clostridioides difficile 630 (Genbank ID: AJP10844.1) and Oceanithermus profundus DSM 14,977 (Genbank ID: ADR35584.1) encoding the MGS enzymes were synthesized by Bioneer Co. (Republic of Korea). For affinity chromatography-mediated protein purification, a Strep tag consisting of a nine residue-long polypeptide was cloned via PCR to the $\mathrm{N}$ - or C-terminal of the MGSs with specific primers (Supplementary Table S1). A six-aspartate tag was also added to the N-terminal of opMGS to increase the solubility of the protein. PrimSTAR Max DNA polymerase (Takara, Japan) was used for the PCR following the manufacturer's protocol. The pETduet-1 vector was linearized with QuickCut NdeI \& KpnI restriction enzymes (Takara, Japan) before ligation with the PCR products using an In-Fusion HD Cloning Kit (Clontech, USA). The constructed vectors were transformed into competent E. coli DH5a (RBC, Taiwan) and selected on LB agar plates supplemented with $100 \mu \mathrm{g} \mathrm{ml}^{-1}$ ampicillin as a marker. The cells were grown in $50 \mathrm{ml} \mathrm{LB}$ medium supplemented with $100 \mu \mathrm{g} \mathrm{ml}{ }^{-1}$ ampicillin at $37^{\circ} \mathrm{C}, 200 \mathrm{rpm}$ in a shaking incubator. The vectors were miniprepped and their sequences were confirmed by DNA sequencing.

Protein expression and purification. The cloned expression vector-transformed E. coli BL21 were selected on LB agar plates and cultivated in $50 \mathrm{ml} \mathrm{LB}$ medium at $37^{\circ} \mathrm{C}, 200 \mathrm{rpm}$, each supplemented with $100 \mu \mathrm{g} \mathrm{ml}^{-1}$ ampicillin to an optical density $(600 \mathrm{~nm})$ of $0.5-0.7$. The cultures were then induced with $80 \mu \mathrm{M}$ isopropyl- $\beta$-thiogalactopyranoside (IPTG) at $20^{\circ} \mathrm{C}, 200 \mathrm{rpm}$ for $19 \mathrm{~h}$. The cells were harvested by centrifugation at $4500 \times g, 4^{\circ} \mathrm{C}$ for $10 \mathrm{~min}$ and stored at $-80^{\circ} \mathrm{C}$ as cell pellets for further uses.

For total protein extraction, the cell pellets were re-suspended in sonication buffer (Biosesang, Korea) and incubated $20 \mathrm{~min}$ on ice. These solutions were sonicated on ice with Ultrasonic Generator $(20 \mathrm{kHz}, 700 \mathrm{~W}$, ULSSO HI-TECH CO., Republic of Korea) setting $50 \%$ power, $2 \mathrm{~s}$ on $/ 3 \mathrm{~s}$ off program until cell lysates were obtained. The cell lysates were centrifuged at $10,000 \times g$ for $15 \mathrm{~min}$ at $4{ }^{\circ} \mathrm{C}$ to discard cell debris. Clear supernatants were applied to affinity chromatography using Strep-Tactin resin (IBA, Germany) according to the manufacturer's recommendations to purify the MGS enzymes ${ }^{23,24}$. Protein Assay Kit (Bio-Rad, USA) was used to quantify the purified proteins.

SDS-PAGE analysis. The insoluble total protein, soluble total protein and purified MGSs from each strain were prepared as samples during the protein purification steps. All samples were diluted to a final concentration of $10 \mu \mathrm{g}$ protein per $16 \mu \mathrm{l}$ and mixed with $4 \mu \mathrm{l}$ of $5 \times$ SDS-PAGE sample buffer (Biosesang, Korea). After boiling $\left(5 \mathrm{~min}, 95^{\circ} \mathrm{C}\right.$ ), the samples were cooled on ice. $20 \mu \mathrm{l}$ of the samples or $7 \mu \mathrm{l}$ of ProSieve Color Protein Marker (Lonza, Swiss) were loaded to each lane of 4-12\% Tris gels (Bio-Rad, USA). Gel electrophoresis was carried out in Tris-glycine-SDS buffer (0.3\% Tris, $1.4 \%$ glycine, $0.1 \%$ SDS (w/v)) at $200 \mathrm{~V}$ using PowerPac Basic Power Supply (Bio-Rad, USA). Coomassie Brilliant Blue G-250 Staining Solution (3 M, USA) was used to stain the gels with microwaves, followed by destaining for $1-2 \mathrm{~h}$ in $30 \%$ methanol $/ 10 \%$ acetic acid $(\mathrm{v} / \mathrm{v})$ destaining buffer with shaking $(50 \mathrm{rpm})$.

MGS activity assay. MGS activity assay using spectrophotometric determination of MG was described previously ${ }^{10,37}$. In more detail, a reaction mixture containing $0.44 \mathrm{ml}$ of $40 \mathrm{mM}$ reaction buffer, $50 \mu \mathrm{l}$ of $30 \mathrm{mM}$ DHAP and $10 \mu \mathrm{l}$ of $10 \mu \mathrm{g} \mathrm{ml}^{-1}$ purified MGS were incubated at $30^{\circ} \mathrm{C}$ for $10 \mathrm{~min}$ in Thermomixer (Eppendorf, USA). After the reaction, $100 \mu \mathrm{l}$ of each reacted solution was transferred to a mixture of $0.9 \mathrm{ml} \mathrm{H}_{2} \mathrm{O}$ and $0.33 \mathrm{ml}$ 2,4-dinitrophenylhydrazine $(0.1 \%(\mathrm{w} / \mathrm{v}) 2,4$-dinitrophenylhydrazine in $2 \mathrm{~N} \mathrm{HCl})$ and were incubated at $30^{\circ} \mathrm{C}$ for $15 \mathrm{~min}$. Finally, $1.67 \mathrm{ml}$ of $(\mathrm{w} / \mathrm{v}) \mathrm{NaOH}$ solution was added and additionally incubated at $30^{\circ} \mathrm{C}$ for $15 \mathrm{~min}$. The optical density at $555 \mathrm{~nm}$ was determined to measure the MG concentration using a molar extinction coefficient of $4.48 \times 10^{4}$.

Buffers for determining the effect of $\mathrm{pH}$ on the MGS activity. The purified enzymes were dialyzed and re-suspended in various buffers ranging from $\mathrm{pH}$ 4-9 to investigate the effect of $\mathrm{pH}$ on the MGS activities and identify the $\mathrm{pH}$ optima. $40 \mathrm{mM}$ succinic acid- $\mathrm{NaOH}$ buffer ( $\mathrm{pH} 4-6$ ), imidazole- $\mathrm{HCl}$ buffer ( $\mathrm{pH} 7$ ), tris(hydroxymethyl)-aminomenthane buffer ( $\mathrm{pH} 8$ ) and glycine- $\mathrm{NaOH}$ buffer ( $\mathrm{pH}$ 9) were used as reaction buffers, from which an optimum buffer and $\mathrm{pH}$ was selected. 
Temperature optimum and thermostability of cdMGS and opMGS. Buffers were first prepared at the temperature of use to minimize $\mathrm{pH}$ change over the different temperatures. In order to determine the optimum temperatures $\left(\mathrm{T}_{\mathrm{opt}}\right.$ ) for the purified MGSs, the substrate in $40 \mathrm{mM}$ imidazole-HCL buffer ( $\mathrm{pH}$ 7) was pre-incubated at desired temperatures $\left(10-90^{\circ} \mathrm{C}\right)$ for $10 \mathrm{~min}$ before the enzyme was added and incubated for $5 \mathrm{~min}$ and the MGS activity assay was carried out. For the thermostability determination, each purified MGS was incubated at different temperatures $\left(30-95^{\circ} \mathrm{C}\right)$ for $15 \mathrm{~min}$ and then immediately cooled on ice ${ }^{5}$. The remaining activity was then measured at $30^{\circ} \mathrm{C}, \mathrm{pH} 7$ as described in the MGS activity assay method.

Molecular modeling. Hexameric enzyme structures of opMGS and tsMGS were homology-modeled based on the crystal structure of the MGS from T. thermophiles HB8 (PDB ID: 1WO8), while that of cdMGS was modeled on the MGS from E. coli (PDB ID: 1EGH) with the Maestro Prime module from Schröedinger (New York, NY, USA) as previously described ${ }^{38,39}$. DHAP structure was created with all possible ionization states via the LigPrep module and docked in the opMGS and cdMGS models via the standard precision mode of the Glide module in the Maestro software ${ }^{40,41}$. The opMGS, tsMGS and cdMGS structures were subjected to Desmond molecular dynamics simulation by creating TIP3P hydration models with OPLS2005 forcefield ${ }^{42}$. The production type was NVT, and the simulation consisted of $400 \mathrm{~ns}$ duration at $358 \mathrm{~K}$ temperature. The trajectories of the enzymes were saved every $400 \mathrm{ps}$, producing 1,000 frames for analysis.

Received: 1 July 2020; Accepted: 11 January 2021

Published online: 28 January 2021

\section{References}

1. Cooper, R. A. \& Anderson, A. The formation and catabolism of methylglyoxal during glycolysis in Escherichia coli. FEBS Lett. 11, 273-276 (1970).

2. Yuan, P. M. \& Gracy, R. W. The conversion of dihydroxyacetone phosphate to methylglyoxal and inorganic phosphate by methylglyoxal synthase. Arch. Biochem. Biophys. 183, 1-6 (1977).

3. Saadat, D. \& Harrison, D. H. Identification of catalytic bases in the active site of Escherichia coli methylglyoxal synthase: cloning, expression, and functional characterization of conserved aspartic acid residues. Biochemistry 37, 10074-10086 (1998).

4. Dickmanns, A. et al. Structural basis for the regulatory interaction of the methylglyoxal synthase MgsA with the carbon flux regulator Crh in Bacillus subtilis. J. Biol. Chem. 293, 5781-5792 (2018).

5. Pazhang, M., Khajeh, K., Asghari, S. M., Falahati, H. \& Naderi-Manesh, H. Cloning, expression, and characterization of a novel methylglyoxal synthase from Thermus sp. strain GH5. Appl. Biochem. Biotech. 162, 1519-1528 (2010).

6. Hopper, D. J. \& Cooper, R. A. The purification and properties of Escherichia coli methylglyoxal synthase. Biochem. J. 128, 321-329 (1972).

7. Cooper, R. A. Methylglyoxal formation during glucose catabolism by Pseudomonas saccharophila. Identification of methylglyoxal synthase. Eur. J. Biochem. 44, 81-86 (1974).

8. Tsai, P. K. \& Gracy, R. W. Isolation and characterization of crystalline methylglyoxal synthetase from Proteus vulgaris. J. Biol. Chem. 251, 364-367 (1976).

9. Huang, K., Rudolph, F. B. \& Bennett, G. N. Characterization of methylglyoxal synthase from Clostridium acetobutylicum ATCC 824 and its use in the formation of 1, 2-propanediol. Appl. Environ. Microb. 65, 3244-3247 (1999).

10. Landmann, J. J. et al. Crh, the paralogue of the phosphocarrier protein HPr, controls the methylglyoxal bypass of glycolysis in Bacillus subtilis. Mol. Microbiol. 82, 770-787 (2011).

11. Murata, K. et al. Characterization of methylglyoxal synthase in Saccharomyces cerevisiae. Biochem. Biophys. Res. Co. 131, 190-198 (1985).

12. Altaras, N. E. \& Cameron, D. C. Metabolic engineering of a 1,2-propanediol pathway in Escherichia coli. Appl. Environ. Microb. 65, 1180-1185 (1999).

13. Bennett, G. N. \& San, K. Y. Microbial formation, biotechnological production and applications of 1,2-propanediol. Appl. Microbiol. Biot. 55, 1-9 (2001).

14. Berrios-Rivera, S. J., San, K. Y. \& Bennett, G. N. The effect of carbon sources and lactate dehydrogenase deletion on 1,2-propanediol production in Escherichia coli. J. Ind. Microbiol. Biot. 30, 34-40 (2003).

15. Bruins, M. E., Janssen, A. E. M. \& Boom, R. M. Thermozymes and their applications. Appl. Biochem. Biotech. 90, 155 (2001).

16. Vieille, C. \& Zeikus, G. J. Hyperthermophilic enzymes: sources, uses, and molecular mechanisms for thermostability. Microbiol. Mol. Biol. R. 65, 1-43 (2001).

17. Basu, S. \& Sen, S. Do homologous thermophilic-mesophilic proteins exhibit similar structures and dynamics at optimal growth temperatures? A molecular dynamics simulation study. J. Chem. Inf. Model. 53, 423-434 (2013).

18. Shimekake, Y., Furuichi, T., Abe, K., Kera, Y. \& Takahashi, S. A novel thermostable D-amino acid oxidase of the thermophilic fungus Rasamsonia emersonii strain YA. Sci. Rep. 9, 11948 (2019).

19. Modarres, H. P., Mofrad, M. R. \& Sanati-Nezhad, A. Protein thermostability engineering. RSC Adv. 6, 115252-115270 (2016).

20. Jo, B. H. et al. Engineering de novo disulfide bond in bacterial alpha-type carbonic anhydrase for thermostable carbon sequestration. Sci. Rep. 6, 29322 (2016).

21. Riedel, T. et al. Genome resequencing of the virulent and multidrug-resistant reference strain Clostridium difficile 630. Genome Announc. 3, e00276 (2015)

22. Miroshnichenko, M. L. et al. Oceanithermus profundus gen. nov., sp. nov., a thermophilic, microaerophilic, facultatively chemolithoheterotrophic bacterium from a deep-sea hydrothermal vent. Int. J. Syst. Evol. Micr. 53, 747-752 (2003).

23. Schmidt, T. G., Koepke, J., Frank, R. \& Skerra, A. Molecular interaction between the Strep-tag affinity peptide and its cognate target, streptavidin. J. Mol. Biol. 255, 753-766 (1996).

24. Schmidt, T. G. \& Skerra, A. The Strep-tag system for one-step purification and high-affinity detection or capturing of proteins. Nat. Protoc. 2, 1528-1535 (2007).

25. Paraskevopoulou, V. \& Falcone, F. H. Polyionic tags as enhancers of protein solubility in recombinant protein expression. Microorganisms 6, 47 (2018).

26. Shaw, K. L., Grimsley, G. R., Yakovlev, G. I., Makarov, A. A. \& Pace, C. N. The effect of net charge on the solubility, activity, and stability of ribonuclease Sa. Protein Sci. 10, 1206-1215 (2001).

27. Jiang, Z. et al. Effects of net charge and the number of positively charged residues on the biological activity of amphipathic alphahelical cationic antimicrobial peptides. Biopolymers 90, 369-383 (2008). 
28. Ray, S. \& Ray, M. Isolation of methylglyoxal synthase from goat liver. J. Biol. Chem. 256, 6230-6233 (1981).

29. Saadat, D. \& Harrison, D. H. The crystal structure of methylglyoxal synthase from Escherichia coli. Structure 7, 309-317 (1999).

30. Marks, G. T., Harris, T. K., Massiah, M. A., Mildvan, A. S. \& Harrison, D. H. Mechanistic implications of methylglyoxal synthase complexed with phosphoglycolohydroxamic acid as observed by X-ray crystallography and NMR spectroscopy. Biochemistry 40, 6805-6818 (2001).

31. Marks, G. T., Susler, M. \& Harrison, D. H. Mutagenic studies on histidine 98 of methylglyoxal synthase: effects on mechanism and conformational change. Biochemistry 43, 3802-3813 (2004).

32. Chan, M. K., Mukund, S., Kletzin, A., Adams, M. W. \& Rees, D. C. Structure of a hyperthermophilic tungstopterin enzyme, aldehyde ferredoxin oxidoreductase. Science 267, 1463 (1995).

33. Thompson, M. J. \& Eisenberg, D. Transproteomic evidence of a loop-deletion mechanism for enhancing protein thermostability. J. Mol. Biol. 290, 595-604 (1999).

34. Karshikoff, A. \& Ladenstein, R. Ion pairs and the thermotolerance of proteins from hyperthermophiles: a 'traffic rule' for hot roads. Trends. Biochem. Sci. 26, 550-557 (2001)

35. Jorda, J. \& Yeates, T. O. Widespread disulfide bonding in proteins from thermophilic archaea. Archaea 2011, 409156 (2011).

36. Beeby, M. et al. The genomics of disulfide bonding and protein stabilization in thermophiles. PLoS Biol. 3, e309-e309 (2005).

37. Phillips, S. A. \& Thornalley, P. J. The formation of methylglyoxal from triose phosphates. Eur. J. Biochem. 212, 101-105 (1993).

38. Lee, H.-S., Park, J., Yoo, Y. J. \& Yeon, Y. J. A novel d-2-hydroxy acid dehydrogenase with high substrate preference for phenylpyruvate originating from lactic acid bacteria: structural analysis on the substrate specificity. Enzyme. Microb. Tech. 125, 37-44 (2019).

39. Prime (Schrödinger, LLC, New York, NY, Release 2018-4).

40. LigPrep (Schrödinger, LLC, New York, NY, Release 2018-4)

41. Glide (Schrödinger, LLC, New York, NY, Release 2018-4).

42. Desmond (Schrödinger, LLC, New York, NY, Release 2018-4)

\section{Acknowledgements}

This research was supported by the C1 Gas Refinery Program through the National Research Foundation of Korea (NRF) and funded by the Ministry of Science and ICT (2015M3D3A1A01064929).

\section{Author contributions}

G.Y.S. designed the work, performed the experiments and wrote the manuscript. H.S.L. designed the work, performed the computer simulations, analyzed the experimental and simulation data, and wrote the manuscript. H.K. helped with validation of data. S.C. helped with validation of data. J.G.N. helped with validation of data. Y.J.Y. conceived and supervised the Project. J.L. conceived and supervised the project. All authors reviewed and approved the manuscript.

\section{Competing interests}

The authors declare no competing interests.

\section{Additional information}

Supplementary Information The online version contains supplementary material available at https://doi. org/10.1038/s41598-021-82078-7.

Correspondence and requests for materials should be addressed to Y.J.Y. or J.L.

Reprints and permissions information is available at www.nature.com/reprints.

Publisher's note Springer Nature remains neutral with regard to jurisdictional claims in published maps and institutional affiliations.

(c) (i) Open Access This article is licensed under a Creative Commons Attribution 4.0 International License, which permits use, sharing, adaptation, distribution and reproduction in any medium or format, as long as you give appropriate credit to the original author(s) and the source, provide a link to the Creative Commons licence, and indicate if changes were made. The images or other third party material in this article are included in the article's Creative Commons licence, unless indicated otherwise in a credit line to the material. If material is not included in the article's Creative Commons licence and your intended use is not permitted by statutory regulation or exceeds the permitted use, you will need to obtain permission directly from the copyright holder. To view a copy of this licence, visit http://creativecommons.org/licenses/by/4.0/.

(C) The Author(s) 2021 